\title{
Electromagnetic FEM studies of disruptions and engineering conse- quences for the power supply and coils design of planned upper divertor at ASDEX Upgrade
}

\author{
M. Teschke*, A. Herrmann, G. Pautasso, T. Vierle, I. Zammuto and the ASDEX Upgrade team
}

\author{
Max Planck Institute for Plasma Physics \\ Boltzmannstr. 2, D-85748 Garching, Germany
}

\begin{abstract}
A new upper divertor is proposed for the ASDEX Upgrade (AUG) tokamak experiment. It is planned to be equipped with internal coils for investigation of alternative magnetic configurations like e.g. „,snowflake“ [1]. Since the coils are close to the plasma, high induced and very stiff voltages are expected during disruption events. Because only very vague analytical estimates of voltages, forces and coupling factors were available, an improvement by the help of Finite Element Method (FEM) was envisaged. Therefore, recorded measurements of currents, plasma position, plasma profile and the geometry were integrated in an electromagnetic simulation as boundary conditions to calculate resulting field distributions during selected AUG disruptions. The time resolution can be better than $100 \mu \mathrm{s}$ and the required computing resources are comparably small due to the assumption of 2D axis-symmetry. The results were compared with magnetic probe measurements integrated into the tokamak ultimately observing good agreement. After this, the FE model was modified including the new divertor to calculate all relevant parameters. The output of these calculations has strong implications for the coil and power supply design: i) The power supply will be protected by a new kind of crowbar to avoid damage of the power supply due to overvoltage and un controlled current and force rise of the coils. The concept of this so called "ripping crowbar" is introduced, which is now under development. ii) The coil cable should be coaxial shaped to monitor isolation faults and to become inherently safe against single-turn shortcuts, identified as destructive fault scenario.
\end{abstract}

Keywords: divertor coils, snowflake, FEM, disruptions, magnetic forces, power supply, crowbar

\section{Introduction}

The scientific goal of the planned upper divertor is to analyze alternative magnetic configurations generated by the divertor coils to reduce the heat load seen by the target plates as shown in Fig. 1 [2]. For a fusion power plant the position of the coils would be chosen in a way that both coils are equal in shape, equal in number of turns, equal in operation coil current amplitudes and opposite in coil current polarity to realize a unique target magnetic configuration.

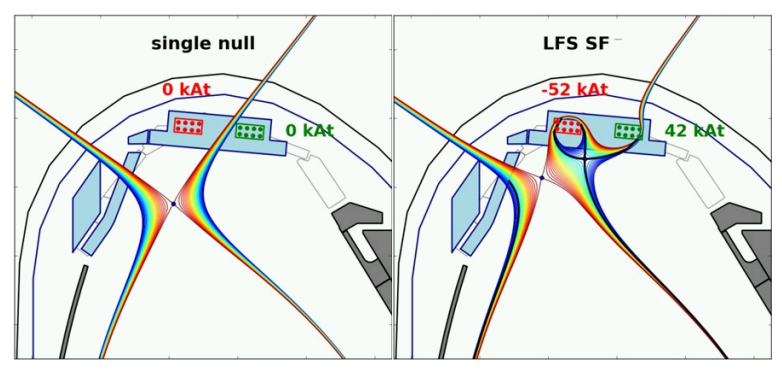

Fig. 1. Magnetic configuration close to the divertor target plates w/o (left) and with divertor coil currents (right)

In this case, both coils can be operated by a single power supply in counter serial connection, as shown in Fig. 2. Coil voltages would almost compensate each other even in high dynamic cases like plasma disruptions. Also the forces on the complex magnetic environment of a tokamak remain small in any case because both magnetic fields compensate each other at far distance (dipole field).

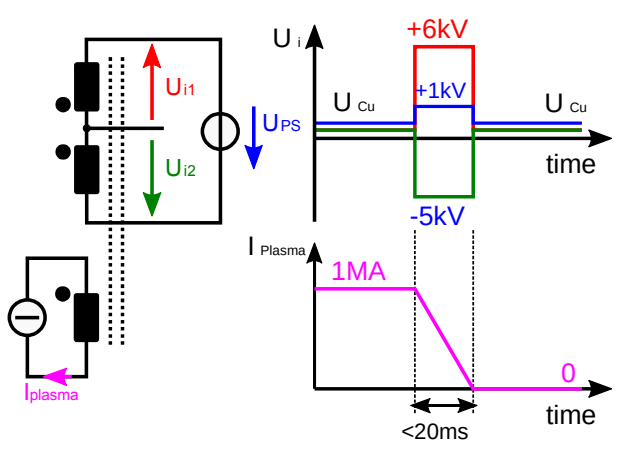

Fig. 2. Coil operation with a single power supply in counter serial connection. Induced voltages during disruptions (red, green) due to strong coupling with the plasma current almost compensate each other.

Nevertheless, as already seen in Fig. 1 the situation is different for a scientific experiment such as AUG. Here, many different magnetic configurations are of interest and they have to be accessible in a simple way, typically by accepting small imbalances in coil currents. For doing so, there exists no other (good) option than connecting the mid-point of both coils to another power supply or inverter providing the requested current difference. This power supply sees the full induced voltage of a single divertor coil during a disruption.

This problem was identified quite early but a proper method to investigate its extent did not exist. We started to think about computational methods to find out $i$ ) how high the induced voltages can really get and what the typical

*author’s email: teschke@ipp.mpg.de 
time evolution is, ii) how strong the magnetic coupling between plasma and coils can be and iii) the consequences of different fault scenarios like full coil shortcut (by power supply faults), single turn short cut (by isolation faults) and under-rated (in voltage) power supplies.

\section{FEM approach}

AUG is in operation since 1991 and consequently a huge database of plasma shots exists. Why not take advantage of it? Thus, the approach presented here is a kind of data processing supported by FEM (see Fig. 3).

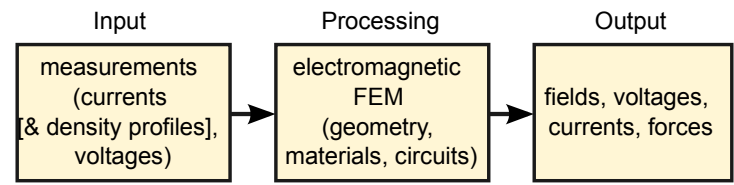

Fig. 3: FEM approach

A quasi-static ${ }^{1}, 2 \mathrm{D}$-axisymmetric electromagnetic FEM simulation is setup with ANSYS MAXWELL V18.2 and the following boundary conditions as input:

- the full, original geometry (w/o divertor coils), including vessel conductivity

- time traces of all active poloidal field coil currents

- time traces of plasma current and position

- the last available reconstruction of plasma current density (before the disruption occurs)

All time traces are taken from a real plasma experiment recording ("shotfile”). Self-consistent, electromagnetic calculations are done for:

- the passive coil structures like the "passive stabilizing loop" (PSL) and the vessel

- the connected power supplies and active parts (switches, diodes, voltage limiters)

- the electric faults to analyze

To simulate a realistic behaviour, these components are connected to an external electric circuit updated for each time step in parallel. The PSL is a quasi-axissymmetric structure consisting of two current loops. A current bridge is connecting the two loops and it forces during vertical displacement events. The resistors avoid floating and too high voltages during disruptions, plasma current quenching (w/o plasma displacement) and (as a drawback) during plasma breakdown. The symmetry is disturbed only in the region of the current bridge. Of course, this is neglected by the 2D simulation (see Fig. 4).

The output of the simulation is a reconstruction of the electromagnetic environment (electric potential and magnetic vector field distribution) for a specific plasma experiment ("plasma shot"). Out of this, electric voltages, currents, current densities, inductances, coupling factors, energy and power distributions, magnetic forces, torques etc. can be computed during the post-processing.

\footnotetext{
${ }^{1}$ Quasi-static approach: electric and magnetic vector potential are treated independently, that means electromagnetic wave propagation is not taken into account.
}

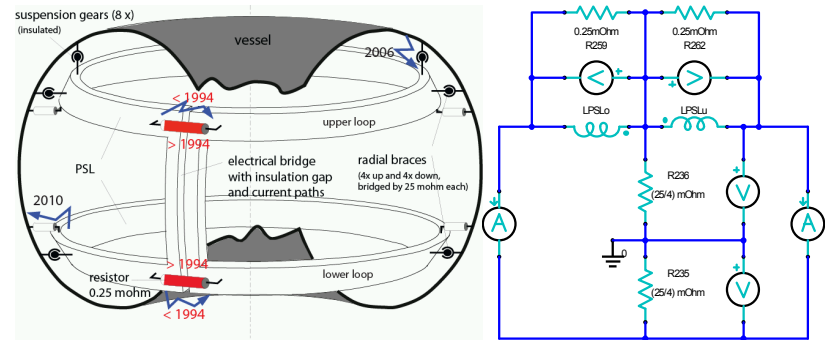

Fig. 4: Left: The PSL geometry embedded into the plasma vessel. Right: The equivalent electric representation. The coupling between the FEM and the electrical circuit is done through the LPSLo/u

Also magnetic probes distributed in the tokamak can be part of the simulated geometry and their output can directly be compared with shotfile data. An example is shown in Fig. 5. At this point, it has to be emphasized that no plasma physics is involved by the calculations. The results are surprisingly realistic due to the fact that all the plasma physics is indirectly involved by the imported plasma current and position traces.
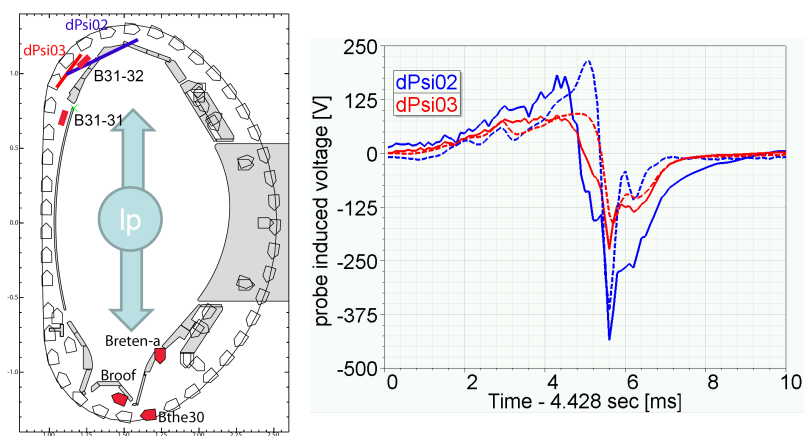

Fig. 5: Magnetics probes installed into the tokamak (left). Comparison of two probe signals (blue: dPsi02, red: dPsi03): simulated (straight curves) and measured (dashed curves) for \#34498. It was compared the probe's raw data (volts instead of magnetic flux)

The only degree of freedom during this setting up process is the plasma current density profile during the disruption after the last available magnetic reconstruction. The influence of this on the simulation results is small unless the current profile is cut by the wall. Halo currents [3] are not explicitly taken into account. For the magnetics it is not relevant if a current is conducted by the vacuum or by a solid wall (the change of decay rate is seen from the plasma current measurement).

After this setting up and validation process, the geometry is modified modeling the target geometry (including the divertor coils). Now, the simulation can be restarted to answer the question "What would have happened if the divertor coils are installed during a specific plasma shot?”

\section{Results}

\subsection{Worst Case Disruption}

An extensive database search was performed to find a "worst case disruption" with the largest $\mathrm{dB} / \mathrm{dt}$ close to the upper divertor. The results are shown in Fig. 6. For every shot, the maximum $\mathrm{dB} / \mathrm{dt}$ of an upper and a lower magnetic probe (compare Fig. 5) is plotted against $\mathrm{x}$ - and $\mathrm{y}$ - 
axis. Dots close to the $\mathrm{x}$-axis disrupted close to the lower divertor and dots close to the y-axis disrupted close to the upper divertor. Shot \#34498 was identified as "worst case shot" for the upper divertor region and chosen to be further analyzed.

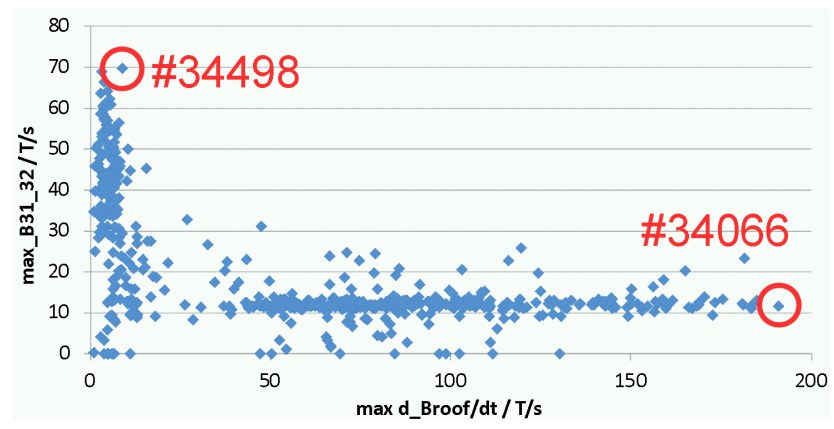

Fig. 6: Database search for worst case disruptions (for the probe positions see Fig. 5).

\subsection{Open loop voltage}

A first outcome of the simulations is a precise information about the requirements from power supply side in terms of output voltage. The open loop voltage of the unconnected divertor coils during the disruption in \#34498 is shown in Fig. 7.
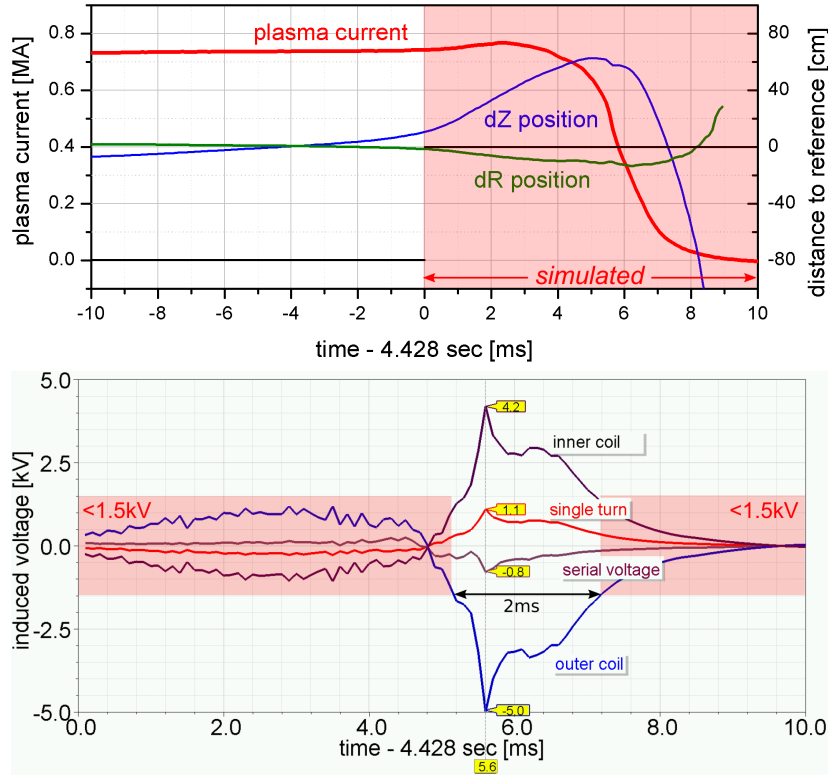

Fig. 7: Top: plasma current and position measurements (= simulation input data) during last $20 \mathrm{~ms}$ of \#34498. Bottom: Calculated divertor coils open loop voltages.

It can be seen that the coil voltage is raised for the first $5 \mathrm{~ms}$ (up to $\pm 1.5 \mathrm{kV}$ per coil) during vertical displacement event of the plasma. For approx. $2 \mathrm{~ms}$ while plasma is closest to the divertor coils (and probably touching the wall) the induced voltage becomes significant (up to +4 /$5 \mathrm{kV}$ per coil). Only for this short period of time, the total (counter-serial) voltage (seen from the main power supply) rises up to $800 \mathrm{~V}$.

The detailed behaviour of each disruption can be different, but the general picture that very high voltage events $(>1.5 \mathrm{kV})$ happen only on the relative short time scale $<2 \mathrm{~ms}$ is true for all analyzed disruptions.

\subsection{Fault scenarios}

Different fault scenarios were analyzed from power supply and coil side (see Fig. 8, left):

(1) Isolation fault (single turn shortcut of a divertor coil = "magnetic shortcut")

(2) Main power supply clamping (=over voltage protection by crowbar activation)

(3) Differential power supply clamping

Furthermore, different protection scenarios were developed and analyzed (see Fig. 8, right):

(4) Use of coaxial cable for the divertor coils and monitoring of common mode potential change to protect against magnetic shortcut

(5) Alternative (quicker!) overvoltage protection of power supplies by the help of varistors (instead or in combination with crowbars)

(6) Alternative overvoltage protection of differential power supply by the help of an intelligent fuse ("ripping crowbar", under development)

(7) Electric decoupling of differential power supply

- Combinations of (4)...(7)

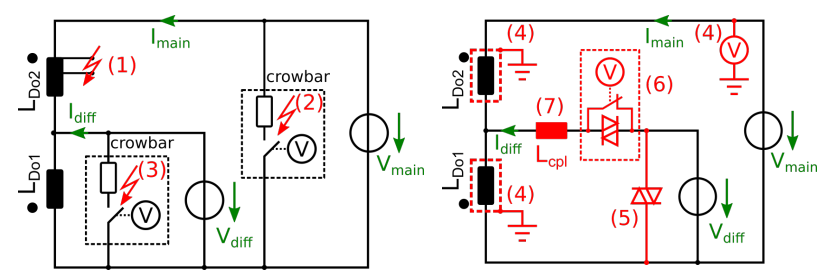

Fig. 8: Analyzed fault scenarios (left) and advanced protection methods (right)

For the first scenario, the evolution of current in and forces on the faulty turn are shown in Fig. 9. An insulation fault of a single turn in one of the divertor coils occurring after $5.5 \mathrm{~ms}(+4.428 \mathrm{sec}$ after plasma breakdown) was simulated when the induced voltage is very close to the maximum (most probable situation). In this case, the current is only limited by ohmic losses (from conductor and arc) and the length of the disruption. Due to the small losses, the decay time constant of the current is much bigger than the total length of the disruption and the very high Lorentz forces (> 20 tons equivalent weight force in vertical direction) decay with the same rate.

Other fusion experiments come to even harder results. For the WEST tokamak recently installed divertor coils, worst case forces of up to $890 \mathrm{kN}$ were expected [4] and they overcome the problem by mounting a robust supporting structure of the coils mechanically independent from the vessel structure. Such solution is not feasible for AUG due to the limited access to the supporting structure of the tokamak. Therefore the new coils have to be mounted inside the vacuum vessel and connected to it. Consequently, we decided that we have to be inherently safe against this fault scenario. Therefore, coaxial cables with an outer shield connected to vessel potential will be used for the design of the divertor coils. In case of an insulation fault, only a ground connection will be the consequence (which can be detected in-situ) which does not result in an uncon- 
trolled current rise for the potential-free (= insulated by a transformer) power supplies.

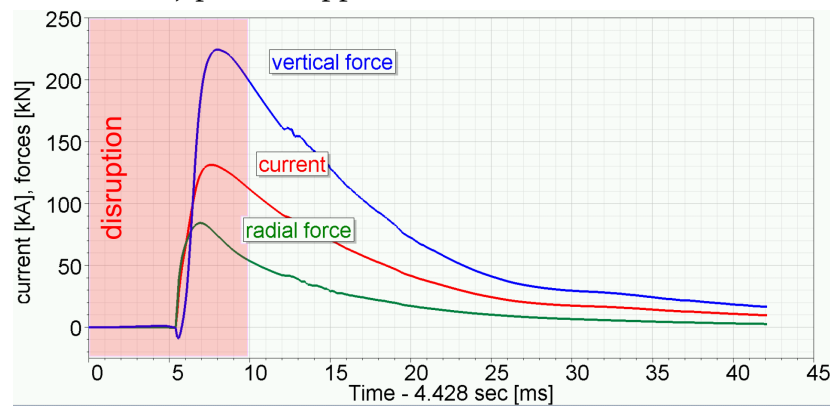

Fig. 9: Current and forces for the single-turn shortcut scenario (see (1) in Fig. 8) of shot \#34498

The results in terms of currents and forces for different scenarios are summarized in 1 . It can be seen that normal operation forces at maximum current and (more important!) maximum current inbalance are in the range of $55 \mathrm{kN}$ during a worst case disruption scenario. For comparison, the vessel weight is around 60 tons $(=600 \mathrm{kN})$.

A second critical scenario is a shortcut or clamping event at the differential power supply side. Here forces of about $300 \mathrm{kN}$ are calculated (= half of the vessel weight) and this is too much for the supporting structure. To solve the problem there are only two options: i) We forbid operation of coils with imbalanced currents. This was decided by scientific staff to be not acceptable. ii) We have to deal with the full high voltage induced by the coils and to avoid that this voltage turns into significant currents (which are related to forces).

In general, this is possible by use of sufficient high voltage power supplies. If the maximum output voltage of the differential power supply is chosen to be bigger than the worst case induced voltage, every disruption event can be kept under control. From a practical point of view, this would be awful solution because one has to provide the very high voltage ( $>5 \mathrm{kV}$ ) at relative high current level (up to $3 \mathrm{kA}$ ) even though the normal operation voltage is very small $(<100 \mathrm{~V})$. The technical solution would be very expensive ( $>>1$ mio€) especially if small current/ field ripple is required.

An alternative solution is the development of an intelligent fuse (named "ripping crowbar") connected in serial between coil and power supply (see (6) in Fig. 8). This device has to actively overtake the full induced voltage at full current for the time of the disruption. The peak power seen from this device is enormous $(5 \mathrm{kV} \cdot 3 \mathrm{kA}=15 \mathrm{MW})$ but the energy can be small $(<100 \mathrm{~kJ})$. As mentioned, this is currently under development (and will be published, soon).

Another compromise could be possible by the combination of two methods: i) Decoupling of the power supply from the load for the short time scale of a disruption by an additional inductance $\mathrm{L}_{\mathrm{cpl}}$ connected in serial (see (7) in Fig. 8). ii) providing as much counter voltage as possible from the power supply side by alternative overvoltage protection with a (powerful) varistor. A comfortable situation arises if the differential power supply voltage is bigger than $1 \mathrm{kV}$. In this case, most of the disruption period of time the current can be kept under control. For only 2 ms (compare Fig. 7) the even higher induced voltage peak has to be overtaken from the serial inductance to limit the current and(!) the current imbalance to an acceptable value. But even for a power supply providing only $500 \mathrm{~V}$, the inductance can be in the range of only some $1 \mathrm{mH}$. This method lives from the observation that disruptions typically provide high $\mathrm{dB} / \mathrm{dt}$ for very short period of time (here $<2 \mathrm{~ms}$ ). If the full high voltage would have to be overtaken for the full disruption period, the $\mathrm{L}_{\mathrm{cpl}}$ value would become unrealistically high $(>>100 \mathrm{mH})$. A drawback of this method is the reduction of bandwidth. E.g. current sweeping capability is reduced. An advantage to the ripping crowbar is the lack of conducting losses $>10 \mathrm{~kW}$ (copper/core losses of $\mathrm{L}_{\mathrm{cpl}}$ neglected). Currently, we see this method as fallback solution, more precise analysis of shotdata is required.

\begin{tabular}{|l|l|l|}
\hline Scenario & $\mathbf{I}_{\mathbf{D} \mathbf{1} / 2}[\mathbf{k A}]$ & $\mathbf{F}_{\mathbf{Z D 0} 1 / 2}[\mathbf{k N}]$ \\
\hline no fault, $\mathrm{I}_{\max }$ & $+13 /-10$ & $+105 /-50$ \\
\hline no fault, open & \pm 0 & $+1.8 /+1.3$ \\
\hline$(1)$ & $+\mathbf{1 3 0 / + 0}$ & $+\mathbf{2 2 5 / + 0}$ \\
\hline$(2)$ & \pm 5 & $+37 /-36$ \\
\hline$(3)$ & $+44 /+0$ & $+308 /+0$ \\
\hline$(5) \mathrm{V}_{\text {clamp }}=1 \mathrm{kV}$ & $+30 /+0$ & $+190 /+0$ \\
\hline$(5) \mathrm{V}_{\text {clamp }}=2.5 \mathrm{kV}$ & $+9 /+0$ & $+50 /+0$ \\
\hline$(6) \mathrm{V}_{\text {clamp }}>5 \mathrm{kV}$ & \pm 0 & \pm 0 \\
\hline$(5+7) \quad \mathrm{V}_{\text {clamp }}=500 \mathrm{~V}, \mathrm{~L}_{\mathrm{cpl}}=0.1 \mathrm{mH}$ & $+9.5 /+0$ & $+65 /+0$ \\
\hline$(5+7) \quad \mathrm{V}_{\text {clamp }}=500 \mathrm{~V}, \mathrm{~L}_{\mathrm{cpl}}=1 \mathrm{mH}$ & $+1.7 /+0$ & $+12 /+0$ \\
\hline
\end{tabular}

Table 1: Coil currents and forces for different scenarios (see Fig. 8). Values add up to the normal operating values $\left(1^{\text {st }}\right.$ and $2^{\text {nd }}$ row). Faults are always induced during period of highest, induced voltage between $5.4 \ldots 5.6 \mathrm{~ms}$ (+4.428 sec after plasma breakdown) of shot \#34498

\section{Final Remarks}

The presented FEM model still provides only some kind of worst case estimate because the plasma is not influenced by additional installations coupled to it and extracting magnetic energy. This influence is assumed to be negligible. It is also not known, how different the disruption behaviour for the new magnetic configurations will be. Another issue not discussed here is the influence of the new coils on plasma breakdown. This is a complex topic we like to publish separately, soon.

Acknowledgements: This work has been carried out within the framework of the EUROfusion Consortium and has received funding from the Euratom research and training programme 2014-2018 and 2019-2020 under grant agreement No 633053. The views and opinions expressed herein do not necessarily reflect those of the European Commission.

\section{References}

[1] A. Herrmann et al, Fus. Eng. Des. 123 (2017) pp. 508-512

[2] T. Lunt et al, Nucl. Mat. Energy 12 (2017), pp. 1037-1042

[3] G. Pautasso et al, Nucl. Fusion 51 (2011) 043010

[4] S. Larroque et al., Fus. Eng. Des. 89 (2014), pp. 1832-183 